\title{
Emollients and smoking: a fire hazard that could be prevented to reduce future deaths
}

Emollients are readily available in prescription and over-the-counter products, and are commonly used in homes and healthcare facilities. These products are not harmful or flammable in isolation, but if they are combined with flames, such as the light of a cigarette, and fabric, they accelerate fires, resulting in burns and deaths. This article describes a Prevent Future Death (PFD) case report of a 74-year-old woman who succumbed to burns in the presence of paraffin-based emollient creams and a lighted match. We highlight the fire risks of such products and the need for greater awareness among the public and healthcare professionals.

Maja K. Bilip ${ }^{1 *}$, Georgia C. Richards²

${ }^{1}$ Oxford Health NHS Foundation Trust, John Radcliffe Hospital, Oxford, OX3 9DU, UK.

${ }^{2}$ Centre for Evidence-Based Medicine, Nuffield Department of Primary Care Health Sciences, University of Oxford, Radcliffe Observatory Quarter, Woodstock Road, Oxford, OX2 6GG, UK.

*corresponding author: Dr Maja K. Bilip, maja.bilip@doctors.net.uk

Word count: 1109

This article is part of the Coroners' Concerns to Prevent Harms series [1]. It discusses a Prevention of Future Death (PFD) report on the fire accelerants properties of emollient products [2].

Products containing emollients, from E45 and Vaseline to Cetraben, are widely available over the counter or by prescription. In the last 12 months (October 2019 to September 2020) more than 15 million items containing emollients were dispensed in England, costing more than £93 million [3]. They come in various formulations, including liquids, creams, and ointments (Box 1), and are used to hydrate the skin. Emollients can contain paraffin, an oily liquid composed of saturated hydrocarbons from petroleum [4], to help form a protective film over the skin and trap moisture. Paraffin is cheaper than alternative agents, such as lanolin or olive oil [5]. If their residue on fabric is exposed to fire (e.g. from the light of a cigarette or stovetop), emollients (both paraffin-free and paraffin-based) can act as accelerants to the fire, and have devastating consequences. It was previously thought that the risk of fire was posed only by emollients containing more than $50 \%$ 
paraffin, but it has been acknowledged that the fire risk cannot be excluded in non-paraffin based emollients [6][7]. Thus, the lack of awareness of the fire risks may cause further harm.

Box 1. EBM facts: Paraffin-based emollients [8]

Formulations

- liquids;

- creams;

- ointments;

- gels;

- dressings;

- sprays.

Ingredients

- paraffin-derived components, which may include:

- white soft paraffin;

- liquid paraffin;

- liquid paraffin light; or

- yellow soft paraffin.

- certain formulations may contain one or more of the following:

- alcohols and acids: benzyl alcohol, cetostearyl alcohol (including cetyl and stearyl alcohol), wool alcohols, isopropyl palmitate, isopropyl myristate, and sorbic acid;

- salts: disodium edetate, magnesium sulfate, sodium lactate, and sodium pidolate;

- preservatives: chlorocresol and phenoxyethanol;

- parabens: hydroxybenzoates;

- emulsifiers: polysorbates or emulsifying wax;

- diols: propylene glycol;

- lanolin or wool fat;

- antimicrobials;

- soya oil; and

o fragrances.

\section{Indications}

- dry skin conditions;

- eczema;

- psoriasis;

- ichthyosis;

- burns;

- pruritus.

\section{Hazards and harms}

- severe and fatal burns due to accelerant properties;

- allergic skin reactions;

- aggravation of acne;

- folliculitis;

- falls, if applied to areas that make surfaces slippery. 
Sixteen deaths from severe burns linked to the accelerant properties of emollients have been reported in the UK (Figure 1). In December 2018, the UK's Medicines and Healthcare products Regulatory Agency (MHRA) reported 11 fatal burn injuries from paraffin-based emollients, and the Fire and Rescue Services reported 50 fire incidents (49 fatal) involving paraffin-based emollients between 2000 and 2018 [7]. Updated data from the MHRA identified five additional fatalities since the 2018 report (Figure 1) [9]. Others have highlighted the fire hazards of using these products, and the need to share information for patients [10][11]. In this Coroners' Concerns to Prevent Harms article, we identify a preventable death from paraffin-based creams [2], and explore how to reduce their harms.

Figure 1: Numbers of deaths from fires or burns relating to emollients, both paraffin-based and paraffin-free, reported to the UK's Medicines and Healthcare products Regulatory Agency (MHRA) Yellow Card Scheme between January 01, 2010 and October 27, 2020. There were 18 reports in total with 16 fatal and two non-fatal outcomes. Data were obtained through a Freedom of Information request to the MHRA [9], and pandas, seaborn, and matplotlib packages in Jupyter Notebooks (Python v3) were used to create the plot. The data and code are openly available on our Github [12].

\section{An accidental death}

In 2019, a 74-year-old woman was pronounced dead in her home after neighbours alerted emergency services to a potential fire. Investigations revealed that the fire had been caused when the deceased used a match to light a cigarette. She had used a paraffin-based emollient cream, but the indication for its use was not mentioned in the coroner's report. She was known to be a heavy smoker, and the paraffin-based cream probably soaked her clothing, acting as an accelerant for the fire. One month before her death, her carers, who were concerned about the risk of fire, had made a safeguarding referral. However, no actions were taken to mitigate harms prior to her death. A low level of carboxyhaemoglobin was identified in the toxicology analysis, and burns were listed as the cause of death. The coroner concluded the inquest as an "accidental death" [2].

During the inquest, the coroner raised several concerns surrounding a lack of awareness of the fire risk of paraffin-based creams by healthcare professionals, carers, and the public (Box 2). The coroner addressed these concerns to six organizations, including: the Department of Health and Social Care, the National Institute for Health and Care Excellence (NICE), Public Health England (PHE), the British Medical Association (BMA), the Care Quality Commission (CQC), and Trent and Dove Social Housing. To date (January 2021), three organizations have responded.

The Department of Health and Social Care responded on January 21, 2020 (due January 17), and described previously seeking advice from the MHRA, which resulted in a Drug Safety Update in December 2018 [7], and an in-depth review by the Commission on Human Medicines (CHM). In its response, the Department also noted that the review by CHM led to two meetings with stakeholders to design resources and methods for their distribution in the form of a toolkit, which was published in July 2020 [13], alongside a press release about the new campaign [14][15]. 
Despite various efforts to share information about the risks, fatalities from fires associated with emollients continue to occur.

Box 2: Coroner's concerns [2]

- Fire investigators reported attending an increasing number of fires involving mostly elderly residents where paraffin-based creams were in use.

- Several creams containing paraffin are readily available in both prescription and over-thecounter products.

- When applied to the skin, they also come into contact with clothing, and may not be washed away in a normal washing cycle, leading to greater contents in clothing.

- If the paraffin causes a fire on clothing, the outcome is highly likely to be lethal, rapidly engulfing the victim in fire, death being primarily due to burns rather than smoke inhalation.

- There is a lack of awareness of the fire risk of such creams by healthcare professionals, carers, and the public. Awareness needs to be raised among healthcare professionals and others who may prescribe or sell paraffin-based products or care for someone using them.

\section{The safe use of emollients}

Emollients are not spontaneously flammable, and they should not be discontinued based on fire risk alone. The dangers arise when emollients are absorbed in clothing, bandages, bedding, or furniture and are combined with an ignition source. People who use large volumes of emollients, apply products often and to large surface areas, those who smoke, and people exposed to open flames (e.g. when smoking or cooking) are at the greatest risk of harm, which appears to be unrecognised in the community.

Successful burn prevention programmes have combined education, product design change, and legislation to prevent harm [16]. Product design change was instigated in 2018 when legislation was implemented requiring all paraffin-containing emollients to display warning labels, and the 2020 MHRA campaign focused on education [7]. In isolation, these measures may not be as effective in preventing harm. Therefore, it is important to implement a multi-pronged approach that educates the public and healthcare professionals, uses product design change in leaflets and warning labels, and critically appraises these methods to ensure evidence-based policy decisions are made going forward.

Current measures to prevent harms may be insufficient. Since this Prevent Future Death (PFD) report was issued by the coroner (November 2019), four further deaths involving emollients have occurred (Figure 1). Other aspects to consider in the prevention of harms from emollient-related fires should include ongoing education, target high-risk groups, and the provision of better evidence related to the fire risks posed by emollients, including effective methods of prevention. While the resources provided by MHRA are freely available, their success in raising awareness amongst the public has not been appraised. Although emollient products contain written information and safety warnings on labels, this may be insufficient as users may not read this information [ref]. In addition, the MHRA campaign does not focus on targeting those at high-risk of emollient related fires. 
It is necessary for high-risk persons to be actively identified at the point of prescribing, dispensing, or purchasing emollients. However, some groups at risk may require additional actions such as referral to a 'stop smoking' service or a fire safety assessment. Relevant government agencies should consider intensifying their efforts to distribute information to patients and highlight the importance of this risk to healthcare providers, which may be lacking in healthcare settings. For this to be successful, a national approach should be considered, that will cascade down to the local level. In a busy clinical environment, alerts and checklists with relevant questions to identify high-risk patients should be considered. The National Pharmacy Association created such a checklist in 2008, but it is unclear how widespread its use is to date [17].

Emollients are the first line treatment for many dermatological conditions, and their importance in managing these conditions should not be overshadowed by their risk of accelerating fires [18]. It should also be noted that other paraffin-containing products, such as some topical antibiotics, may pose a similar risk, and preventative measures should be considered while ensuring the continued safe use of these products. While efforts are being made to raise public awareness of such harms through the media [19-23], actions to prevent future deaths are needed. For informed consent to occur, patients must be made aware of the risks, and healthcare professionals recommending or prescribing emollients must ensure patients are provided with this information. The public should also be encouraged to report harms to the Yellow Card Scheme, to ensure accurate monitoring of suspected harms. To date, third party organisations concerned with dermatological conditions and burns have not played a major role in educating the public about the risks of emollient related fires. These organisations are experienced and well placed to spearhead a collaborative effort to raise awareness and prevent harms.

\section{Declarations of interests}

MKB has no interests to declare. GCR is financially supported by the National Institute of Health Research (NIHR) School for Primary Care Research (SPCR), the Naji Foundation, and the Rotary Foundation to study for a Doctor of Philosophy (DPhil/PhD) at the University of Oxford. GCR is an Associate Editor of BMJ Evidence Based Medicine, founder of the Coroners' Concerns to Prevent Harms series, and is developing the https://preventabledeathstracker.net/. The views expressed are those of the authors and not necessarily those of the NHS, the NIHR, or the Department of Health and Social Care.

\section{References}

1 Richards GC, Aronson JK, Heneghan C. Coroners' concerns to prevent harms: A series of coroners' case reports to serve patient safety and educate the public, clinicians and policy-makers. BMJ Evidence-Based Med. 2020. doi:10.1136/bmjebm-2020-111567

2 M. J. Maureen Milton | Courts and Tribunals Judiciary. https://www.judiciary.uk/publications/maureen-milton/ (accessed 20 Jan 2021). 
3 Datalab. Analyse - Emollients 13.2.1 \& Emollients 21.22. OpenPrescribing.net.Analyse | OpenPrescribing.

https://openprescribing.net/analyse/\#org=CCG\&numlds=13.2.1,21.22\&denom=nothing\&s electedTab=summary (accessed 20 Jan 2021).

4 Sharif $F$, Crushell E, O'Driscoll K, et al. Liquid paraffin: A reappraisal of its role in the treatment of constipation. Arch. Dis. Child. 2001. doi:10.1136/adc.85.2.121

5 Rawlings A V., Lombard KJ. A review on the extensive skin benefits of mineral oil. Int. J. Cosmet. Sci. 2012. doi:10.1111/j.1468-2494.2012.00752.x

$6 \quad$ Hall S, Franklin L, Bull J, et al. The flammability of textiles when contaminated with paraffin base products. Fire Saf J 2019;104:109-16. doi:10.1016/j.firesaf.2019.01.003

$7 \quad$ MHRA. Emollients: new information about risk of severe and fatal burns with paraffincontaining and paraffin-free emollients - GOV.UK. https://www.gov.uk/drug-safetyupdate/emollients-new-information-about-risk-of-severe-and-fatal-burns-with-paraffincontaining-and-paraffin-free-emollients (accessed 20 Jan 2021).

8 NICE. EMOLLIENT CREAMS AND OINTMENTS, PARAFFIN-CONTAINING | Medicinal forms | BNF content published by NICE. https://bnf.nice.org.uk/medicinal-forms/emollientcreams-and-ointments-paraffin-containing.html (accessed 20 Jan 2021).

$9 \quad$ MHRA. Deaths and adverse events from fires caused by emollient skin creams - a Freedom of Information request to Medicines and Healthcare products Regulatory Agency - WhatDoTheyKnow.

https://www.whatdotheyknow.com/request/deaths_and_adverse_events_from_f (accessed 20 Jan 2021).

10 Shokrollahi K. Paraffin-based ointments and fire hazard: understanding the problem, navigating the media and currently available downloadable patient information. Scars, Burn Heal 2017;3:2059513117704932. doi:10.1177/2059513117704932

11 Robin E. Ferner, Craig Easton et al. Deaths from Medicines: A Systematic Analysis of Coroners' Reports to Prevent Future Deaths | SpringerLink. https://link.springer.com/article/10.1007\%2Fs40264-017-05880?fbclid=IwAR1eXs8HMyg_Yz773izqJpCkBo4LfmFc_WpvTYNyJkLwEXyD3ZoMEvNjvW E (accessed 25 Jan 2021).

12 Richards G. pfd_emollients. Github Reports of fires or burns relating to emollients, both paraffin-based and paraffin-free, reported to the UK's MHRA Yellow Card Scheme. https://github.com/georgiarichards/pfd_emollients (accessed 20 Jan 2021).

13 MHRA. Safe use of emollient skin creams to treat dry skin conditions - GOV.UK. https://www.gov.uk/guidance/safe-use-of-emollient-skin-creams-to-treat-dry-skinconditions (accessed 20 Jan 2021).

14 MHRA. Emollients and risk of severe and fatal burns: new resources available - GOV.UK. https://www.gov.uk/drug-safety-update/emollients-and-risk-of-severe-and-fatal-burnsnew-resources-available (accessed 20 Jan 2021).

15 MHRA. Skin creams dried on fabric can lead to fire deaths - GOV.UK. 
https://www.gov.uk/government/news/skin-creams-dried-on-fabric-can-lead-to-fire-deaths (accessed 20 Jan 2021).

16 Liao CC, Rossignol AMK. Landmarks in burn prevention. Burns. 2000;26:422-34. doi:10.1016/S0305-4179(00)00026-7

17 National Pharmacy Association. SOP: Supplying Paraffin-Based and Paraffin-Free Products - NPA » NPA. 2008.https://www.npa.co.uk/information-and-guidance/supplyingparaffin-based-skin-products/ (accessed 22 Feb 2021).

18 British Association of Dermatologists. BAD statement on fire risk associated with emollient use. 2020.https://www.skinhealthinfo.org.uk/bad-statement-on-fire-riskassociated-with-emollient-use/

19 Sheppard C. Which skin creams contain paraffin and what conditions is it used to treat? From eczema to psoriasis. The Sun. https://www.thesun.co.uk/fabulous/5557053/whichskin-creams-contain-paraffin-and-what-conditions-does-it-treat/ (accessed 20 Jan 2021).

20 Speare-Cole R. Woman, 83, dies after accidentally setting herself on fire while wearing flammable skin cream | London Evening Standard | Evening Standard.

https://www.standard.co.uk/news/uk/woman-83-dies-fire-emollient-moisturiser-flammablea4511896.html (accessed 20 Jan 2021).

21 Robinson B GA. Skin creams containing paraffin linked to fire deaths - BBC News. https://www.bbc.co.uk/news/uk-39308748 (accessed 20 Jan 2021).

22 Waldron B. Smoker died in flat fire after dropping lit match into her lap - Derbyshire Live. https://www.derbytelegraph.co.uk/burton/smoker-died-fire-burton-milton-3558038 (accessed 20 Jan 2021).

23 Staffordshire Fire and Rescue Service. Nephew urges caution when using emollient creams on anniversary of aunt's death in fire.

https://www.staffordshirefire.gov.uk/news/latest-news/nephew-urges-caution-when-usingemollient-creams-on-anniversary-of-aunts-death-in-fire/ (accessed 20 Jan 2021). 